Military Technical College

Kobry Elkobbah,

Cairo, Egypt

May 29-31,2012 $6^{\text {th }}$ International Conference on

Mathematics and Engineering

Physics (ICMEP-6)

\title{
Some Characterizations of The Skew Normal Distribution Using Conditional Moments
}

\author{
M.Gharib ${ }^{a}$ and A. Sharawy ${ }^{b}$ \\ ${ }^{a}$ Mathematics Department, Faculty of Science, Ain Shams University, Cairo, Egypt. \\ ${ }^{\mathrm{b}}$ Mathematics Department,Faculty of Science(men), Al-Azhar University, Cairo, Egypt.
}

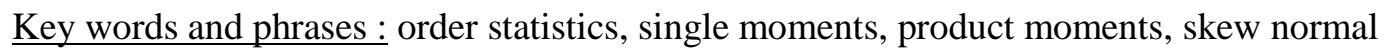

distribution, characterization.

\begin{abstract}
$\underline{\text { Abstract }}$
Very few researchers have tackled the characterization problem of the skew nor- mal distribution due perhaps to its mathematical tractability. In this article, some new characterization results for the skew normal distribution based on conditional moments have been obtained. The results specialized to the standard normal distri- bution. Some consequences and discussions are, also given in this context.
\end{abstract}

\section{1- Introduction}

The skew-normal distribution was first introduced by O'Hagan and Leonard (1976) as a prior distribution for estimating a normal location parameter.

The skew-normal distribution and its variations have been discussed by several au- thors including Azzalini (1985, 1986), Henze (1986), Azzalini and Dalla Valle (1996), Branco and Dey (2001), Loperfido (2001), Arnold and Beaver (2002), Balakrishnan (2002), and Azzalini and Chiogna (2004) and others for a comprehensive survey of de- velopments on skew-normal distribution and its multivariate form see Azzalini (2005).

A random variable $X$ is said to have a standard skew-normal distribution with parameter $\lambda \in \mathbb{R}$, denoted by $X \sim \mathrm{SN}(\lambda)$, if its probability density function (pdf) is:

$f(x, \lambda)=2 \Phi(\lambda x) \phi(x)=\frac{1}{\pi} e^{-\frac{x^{2}}{2}} \int_{-\infty}^{\lambda x} e^{-\frac{t^{2}}{2}} d t . \quad \lambda \in \mathbb{R},-\infty<x<\infty$

The cumulative distribution function (cdf ) of $\mathrm{SN}(\lambda)$ is given by: 
Military Technical College

Kobry Elkobbah,

Cairo, Egypt

May 29-31,2012 $6^{\text {th }}$ International Conference on Mathematics and Engineering

Physics (ICMEP-6)

$F(x, \lambda)=2 \int_{-\infty}^{x} \Phi(\lambda x) \phi(u) d u=\int_{-\infty}^{x} \frac{1}{\pi} \mathrm{e}^{-\frac{\mathrm{u}^{2}}{2}} \int_{-\infty}^{\lambda \mathrm{u}} \mathrm{e}^{-\frac{\mathrm{t}^{2}}{\mathrm{z}}} \mathrm{dt} \mathrm{du}$.

where $\phi($.$) and \Phi($.$) denote the standard normal pdf and cdf, respectively.$

Now, we shall give some definitions that are needed in the sequel.

Definition 1 Let $X_{1}, X_{2}, \ldots, X_{n}$ be independent random variables having skew normal pdf $f(x, \lambda)$ and cdf $F(x, \lambda)$. Let $\mathrm{X}_{1: \mathrm{n}}<\mathrm{X}_{2: \mathrm{n}}<\ldots<\mathrm{X}_{\mathrm{n}: \mathrm{n}}$ denote the corresponding order statistics. Then the pdf of $X_{r i n}, 1 \leq \mathrm{r} \leq \mathrm{n}$ and the joint pdf of $\left(X_{r i n}, X_{\sin }\right)$ can be written as ( Arnold et al. (2008)):

$f_{r n n}(x, \lambda)=C_{r n n}(F(x, \lambda))^{r-1} f(x, \lambda)(1-F(x, \lambda))^{n-r}, \quad 1 \leq r \leq n$

$f_{r: n}(x, y, \lambda)=C_{r i s i n}(F(x, \lambda))^{r-1} f(x, \lambda)(F(y, \lambda)-F(x, \lambda))^{8-r-1} f(y, \lambda)(1-F(y, \lambda))^{n-s}$,

$1 \leq r<s \leq n$

where $C_{r m n}=\frac{n !}{(r-1) !(n-r) !} \quad, \quad C_{r, s+n}=\frac{n !}{(r-1) !(s-r-1) !(n-s) !}$

Definition 2 Let $n \in \mathbb{R}, k \geq 1, m_{1}, m_{2}, \ldots, m_{n-1} \in \mathbb{R}, M_{r}=\sum_{j=r}^{n-1} m_{i}, 1 \leq r \leq n-1$, and let $\bar{m}=\left(m_{1}, m_{2}, \ldots, m_{n-1}\right), m_{1}=m_{1}=\ldots=m_{n-1}=m, m \neq-1$. The pdf of the $r^{\text {th }}$ generalized order statistics (gos) (Kamps 1995) is given by:

$f_{r, n, \bar{m}, k}(x, \lambda)=\frac{d_{r-1}}{(r-1) !}(1-F(x, \lambda))^{n-r+k+M_{r}-1} f(x, \lambda)\left(\frac{1-(1-F(x, \lambda))^{m+1}}{m+1}\right)^{r-1}, 1 \leq r \leq n$

and the joint pdf of the $\mathrm{r}^{\text {th }}$ and the $\mathrm{s}^{\mathrm{th}}$ gos as:

$f_{r, s, n, \bar{m}, k}(x, y, \lambda)=\frac{d_{s-1}}{(r-1) !(s-r-1) !}(1-F(x, \lambda))^{m} f(x, \lambda)\left(\frac{1-(1-F(x, \lambda))^{m+1}}{m+1}\right)^{r-1} \times$
$(1-F(y, \lambda))^{n-s+k+M_{s}-1} f(y, \lambda)\left[\frac{(1-F(x, \lambda))^{m+1}-(1-F(y, \lambda))^{m+1}}{m+1}\right]^{s-r-1}, 1 \leq r<s \leq n$

where $d_{r i n}=\prod_{i=1}^{r} k+n-i+M_{i} \geq 1$ 
Military Technical College

Kobry Elkobbah,

Cairo, Egypt

May 29-31,2012

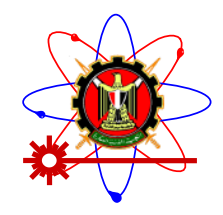

$6^{\text {th }}$ International Conference on Mathematics and Engineering

Physics (ICMEP-6)

Let us denote the $\mathrm{k}^{\mathrm{th}}$ single moment $E\left(X_{r i n}^{k}\right)$ of $X_{r i n}$ by $\mu_{r i n}^{(k)}$ and the product moment $E\left(X_{r i n} X_{\sin }\right)$ of $X_{r i n}$ and $X_{\sin }$ by $\mu_{r, s i n}$.

\section{2- The Results.}

We shall state the main results concerning characterization of the $S N(\lambda)$ distribution.

\section{Theorem 1}

Let $\mathrm{X}$ be a continuous random variable with $\operatorname{cdf} F($ ) , survival function $\bar{F}(\mathrm{)}$, pdf $f($.$) , failure$ rate $h($.$) , finite mean \mu$. Then $\mathrm{X}$ has a skew normal distribution $S N(\lambda)$ with mean $\mu$ if and only if.

$$
\begin{aligned}
& E\left(X^{2 k} \mid X>y\right)=(2 k-1) !+ \\
& \frac{1}{F(y, \lambda)} \sum_{i=1}^{k} \frac{(2 k-1) ! n}{(2 i-1) !}\left(y^{2 i-1} f(y, \lambda)+\frac{2 \lambda}{\sqrt{2 \pi}} \sum_{j=1}^{i} \frac{(2 i-1) ! n}{(2 j-1) !} \frac{y^{2 j-2} \phi\left(y \sqrt{1+\lambda^{2}}\right)}{(1+\lambda)^{2 i-j+1}}\right)
\end{aligned}
$$

with $\mathrm{k}=1.2 .3 \ldots$,

or

$$
\begin{aligned}
& E\left(X^{2 k} \mid X>y\right)= \\
& \left(\frac{1}{\bar{F}(y, \lambda)} \sum_{i=0}^{k} \frac{(2 k) ! n}{(2 i) ! !}\right)\left(y^{2 i} f(y, \lambda)+\frac{(2 i-1) ! !\left(-y \sqrt{1+\lambda^{2}}\right)}{(1+\lambda)^{i+1}}\right)+ \\
& \left(\frac{1}{F(y, \lambda)} \sum_{i=0}^{k} \frac{(2 k) ! n}{(2 i) ! !}\right) \frac{2 \lambda}{\sqrt{2 \pi}} \sum_{j=1}^{i} \frac{(2 i-1) ! n}{(2 j-1) ! n} \frac{y^{2 j-1} \phi\left(y \sqrt{1+\lambda^{2}}\right)}{(1+\lambda)^{i-j+1}}
\end{aligned}
$$

\section{$\underline{\text { Remarks }}$}

(1) From Theorem 1, for $\mathrm{k}=0$, (2.2) reduces to: 
Military Technical College

Kobry Elkobbah,

Cairo, Egypt

May 29-31,2012 $6^{\text {th }}$ International Conference on Mathematics and Engineering

Physics (ICMEP-6)

$E(X \mid X>y)=h(y)+\mu \frac{\Phi\left(-y \sqrt{1+\lambda^{2}}\right)}{\bar{F}(y, \lambda)}$.

The " only if" part of (2.3) in this case can be proved simply as follows:

If (2.4) is true, then:

$E(X \mid X>y)=\frac{1}{\bar{F}(y, \lambda)} \int_{y}^{\infty} x f(x, \lambda) d x=h(y, \lambda)+\mu \frac{\Phi\left(-y \sqrt{1+\lambda^{2}}\right)}{\bar{F}(y, \lambda)}$.

Then we have:

$\int_{y}^{\infty} x f(x, \lambda) d x=f(y, \lambda)+\mu \Phi\left(-y \sqrt{1+\lambda^{2}}\right)$

Differentiating both sides with respect to , we obtain:

$-y f(x, \lambda)=2 \lambda \phi(\lambda y) \phi(y)+2 \Phi(\lambda y) \phi(y)(-y)-\mu \phi\left(y \sqrt{1+\lambda^{2}}\right) \sqrt{1+\lambda^{2}}$

Or, $-y f(x, \lambda)=\frac{2 \lambda}{\sqrt{2 \pi}} \phi\left(y \sqrt{1+\lambda^{2}}\right)+2 \Phi(\lambda y) \phi(y)(-y)-\mu \phi\left(y \sqrt{1+\lambda^{2}}\right) \sqrt{1+\lambda^{2}}$

Or, $-y f(x, \lambda)=2 \Phi(\lambda y) \phi(y)(-y)$

Or, $f(x, \lambda)=2 \Phi(\lambda y) \phi(y)$

which is the pdf of the skew normal distribution $\operatorname{SN}(\lambda)$.

(2) Theorem 1 reducedto the case of the standard normal distribution by putting $\lambda=0$.

\section{Theorem 2}

Let $X$ be a random variable having pdf $f(x)$ and $\operatorname{cdf} F(x)$. Then $X$ have a skew normal distribution $S N(\lambda)$ if and only if

$E\left(X_{s i n}^{k} \mid X_{r i n}=x\right)=x^{k}+\frac{(n-r) !}{(1-F(x, \lambda))^{n-r}} \eta_{s}(x, \lambda), \mid 1 \leq r<s \leq n$,

where: 
Military Technical College

Kobry Elkobbah,

Cairo, Egypt

May 29-31,2012 $6^{\text {th }}$ International Conference on Mathematics and Engineering

Physics (ICMEP-6)

$\eta_{s}(x, \lambda)=$

$\sum_{j=r+1}^{s} k \int_{x}^{\infty} y^{k-1} \frac{\left\lfloor\left[\left(1-\int_{-\infty}^{y} \frac{1}{\pi} e^{-\frac{x^{2}}{2}} \int_{-\infty}^{\lambda x} e^{-\frac{t^{2}}{2}} d t d x\right)^{(n-j=1)}\right] \psi_{1}(x, y, \lambda)\right\rfloor}{(n-j+1) !(j-r-1) !} d y$

and

$\psi_{1}(x, y, \lambda)=$

$\left[\left(\int_{-\infty}^{y} \frac{1}{\pi} e^{-\frac{x^{2}}{2}} \int_{-\infty}^{\lambda x} e^{-\frac{t^{2}}{2}} d t d x-\int_{-\infty}^{x} \frac{1}{\pi} e^{-\frac{u^{2}}{2}} \int_{-\infty}^{\lambda u} e^{-\frac{t^{2}}{2}} d t d u\right)^{(j-r-1)}\right]$

\section{Remarks:}

(1) From Theorem 2, at $s=r+1$, we have:

$E\left(X_{r+1 ; n}^{k} \mid X_{r i n}=x\right)=x^{k}+\frac{1}{(1-F(x, \lambda))^{n-r}} \eta(x, \lambda)$

$\mathrm{k}=1,2,3, \ldots$

where $\quad \eta(x, \lambda)=k \int_{x}^{\infty} y^{k-1}\left(1-\int_{-\infty}^{y} \frac{1}{\pi !} e^{-\frac{x^{2}}{2}} \int_{-\infty}^{\lambda x} e^{-\frac{t^{2}}{2}} d t d x\right)^{[n-r)} d y$.

(2) The result (2.4) reduces to the standard normal distribution case if $\lambda=0$.

i.e.

$E\left(X_{\sin }^{k} \mid X_{r i n}=x\right)=x^{k}+\frac{(n-r) !}{(1-F(x, \lambda))^{n-r}} \eta_{s}(x), \quad 1 \leq r<s \leq n$

where 
Military Technical College

Kobry Elkobbah,

Cairo, Egypt

May 29-31,2012

$\eta_{s}(x)=\sum_{j=r+1}^{s} k \int_{x}^{\infty} y^{k-1} \frac{\left\lfloor\left[\left(1-\int_{-\infty}^{y} \frac{1}{\sqrt{2 \pi}} e^{-\frac{x^{2}}{2}} d x\right)^{(n-j+1)}\right] \psi_{1}(x, y)\right\rfloor}{(n-j+1) !(j-r-1) !} d y$,

and

$\psi_{1}(x, y)=\left[\left(\int_{-\infty}^{y} \frac{1}{\sqrt{2 \pi}} e^{-\frac{x^{2}}{2}} d x-\int_{-\infty}^{x} \frac{1}{\sqrt{2 \pi}} e^{-\frac{u^{2}}{2}} d u\right)^{(j-r-1)}\right]$.

\section{Theorem 3}

Let $\mathrm{X}$ be a random variable having pdf $f(x)$ and $\operatorname{cdf} F(x)$. Then $\mathrm{X}$ have a skew normal distribution $\mathrm{SN}(\lambda)$ if and only if

$$
\begin{aligned}
& E\left(x_{s: n_{2}, \bar{m}_{l}, k}^{k} \mid X_{r i n_{2}, \bar{m}, k}=x\right)= \\
& x^{k}+\left(\frac{[(m+1)(n-s-1)+k]}{(m+1)}\right) ! \frac{\prod_{i=r+1}^{s}[(m+1)(n-r-1)+k](m+1)^{s-r}}{(1-F(x, \lambda))^{(m+1)(n-r-1)+k}} \gamma_{s}(x, \lambda),
\end{aligned}
$$

$1 \leq r<s \leq n$

where

$\gamma_{s}(x, \lambda)=\sum_{j=r+1}^{s} k \int_{x}^{\infty} y^{k-1} \frac{\left\lfloor\left[\left(1-\int_{-\infty}^{y} \frac{1}{\pi} e^{\left.\left.-\frac{x^{2}}{2} \int_{-\infty}^{\lambda x} e^{-\frac{t^{2}}{2}} d t d x\right)^{(m+1)(n-j)+k}\right]} \psi_{1}(x, y, \lambda) \mid\right.\right.\right.}{\left(\frac{[(m+1)(n-j)+k]}{(m+1)}\right) !(j-r-1) !} d y$.

and

$$
\begin{aligned}
& \psi_{m}(x, y, \lambda) \\
& =\left[\left(1-\int_{-\infty}^{x} \frac{1}{\pi} e^{-\frac{u^{2}}{2}} \int_{-\infty}^{\lambda u} e^{-\frac{t^{2}}{2}} d t d u\right)^{m+1}-\left(1-\int_{-\infty}^{y} \frac{1}{\pi} e^{-\frac{x^{2}}{2}} \int_{-\infty}^{\lambda x} e^{-\frac{t^{2}}{2}} d t d x\right)^{m+1}\right]^{(j-r-1)}
\end{aligned}
$$

\section{$\underline{\text { Remarks. }}$}

(1) At $s=r+1,(2.7)$, reduces to 
Military Technical College

Kobry Elkobbah,

Cairo, Egypt

May 29-31,2012 $6^{\text {th }}$ International Conference on Mathematics and Engineering

Physics (ICMEP-6)

$E\left(X_{r+1 ; n, \bar{m}, k}^{k} \mid X_{r i n, \bar{m}, k}=x\right)=x^{k}+\frac{1}{(1-F(x, \lambda))^{(m+1)(n-r-1)+k}} \gamma(x, \lambda), \quad k=1,2,3, \ldots$

where $\quad \gamma(x, \lambda)=k \int_{x}^{\infty} y^{k-1}\left(1-\int_{-\infty}^{y} \frac{1}{\pi} e^{-\frac{x^{2}}{2}} \int_{-\infty}^{\lambda x} e^{-\frac{t^{2}}{2}} d t d x\right)^{[m+1)(n-r-1)} d y$.

(2) Also (2.7) reduces to the standard normal distribution case if $\lambda=0$.

i.e.

$$
\begin{aligned}
& E\left(X_{s i n, \bar{m}, k}^{k} \mid X_{r i n, \bar{m}, k}=x\right)= \\
& \quad x^{k}+\left(\frac{[(m+1)(n-s-1)+k]}{(m+1)}\right) ! \frac{\prod_{i=r+1}^{s}[(m+1)(n-r-1)+k](m+1)^{s-r}}{(1-F(x))^{(m+1)(n-r-1)+k}} \gamma_{s}(x),
\end{aligned}
$$

$1 \leq r<s \leq n$

where

$\gamma_{s}(x)=\sum_{j=r+1}^{s} k \int_{x}^{\infty} y^{k-1} \frac{\left|\left[\left(1-\int_{-\infty}^{y} \frac{1}{\sqrt{2 \pi}} e^{-\frac{x^{2}}{2}} d x\right)^{(m+1)(n-j)+k}\right] \psi_{1}(x, y)\right|}{\left(\frac{[(m+1)(n-j)+k]}{(m+1)}\right) !(j-r-1) !} d y$

and

$\psi_{m}(x, y)=\left[\left(1-\int_{-\infty}^{x} \frac{1}{\sqrt{2 \pi}} e^{-\frac{u^{2}}{2}} d u\right)^{m+1}-\left(1-\int_{-\infty}^{y} \frac{1}{\sqrt{2 \pi}} e^{-\frac{x^{2}}{2}} d x\right)^{m+1}\right]^{(j-r-1)}$

\section{3- The proofs}

\section{proof of theorem 1}

\section{Necessity.}

We have

$$
E\left(X^{k} \mid X>y\right)=\frac{1}{\bar{F}(y, \lambda)} \int_{y}^{\infty} x^{k} f(x, \lambda) d x
$$


Military Technical College

Kobry Elkobbah,

Cairo, Egypt

May 29-31,2012

$$
\begin{aligned}
& =\frac{2}{\bar{F}(y, \lambda)} \int_{y}^{\infty} x^{k} \Phi(\lambda x) \phi(x) d \\
& =\frac{-2}{\bar{F}(y, \lambda)} \int_{y}^{\infty} x^{k-1} \Phi(\lambda x) d \phi(x)
\end{aligned}
$$

$6^{\text {th }}$ International Conference on Mathematics and Engineering

Physics (ICMEP-6)

Integrating by parts we get

$E\left(X^{k} \mid X>y\right)=\frac{2 y^{k-1} \Phi(\lambda y) \phi(y)}{\bar{F}(y, \lambda)}+\frac{2(k-1) \int_{y}^{\infty} x^{k-2} \Phi(X x) \phi(x) d x}{\bar{F}(y, \lambda)}+$

$\frac{2 \lambda \int_{y}^{\infty} x^{k-1} \phi(\lambda x) \phi(x) d x}{\bar{F}(y, \lambda)}$

$=y^{k-1} h(y, \lambda)+(k-1) E\left(X^{k-2} \mid X>y\right)+\frac{2 \lambda}{\bar{F}(y, \lambda)} \int_{y}^{\infty} x^{k-1} \phi(\lambda x) \phi(x) d x$

Let

$\alpha(y, \lambda)=2 \lambda \int_{y}^{\infty} x^{k-1} \emptyset(\lambda x) \phi(x) d x=\frac{\lambda}{\pi} \int_{y}^{\infty} x^{k-1} e^{-\frac{1}{2}\left(1+\lambda^{2}\right) x^{2}} d x$

Put $z=x^{2}$ we get

$\alpha(y, \lambda)=\frac{\lambda}{2 \pi} \int_{y^{2}}^{\infty} z^{\frac{k-2}{2}} e^{-\frac{1}{2}\left(1+\lambda^{2}\right) z} d z$

For $=2 r$, we have

$\alpha_{1}(y, \lambda)=\frac{\lambda}{2 \pi} \int_{y^{2}}^{\infty} z^{r-1} e^{-\frac{1}{2}\left(1+\lambda^{2}\right) z} d z$

Integrating by parts we get 
Military Technical College

Kobry Elkobbah,

Cairo, Egypt

May 29-31,2012

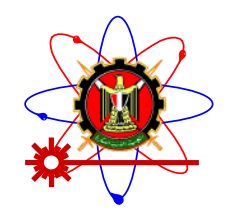

$6^{\text {th }}$ International Conference on Mathematics and Engineering

Physics (ICMEP-6)

$$
\alpha_{1}(y, \lambda)=\left(\frac{k-2}{2}\right) ! \mu \phi\left(y \sqrt{1+\lambda^{2}}\right) \sum_{i=0}^{\frac{k-2}{2}} \frac{2^{i} y^{k-2-2 i}}{\left(\frac{k-2-2 i}{2}\right) !\left(1+\lambda^{2}\right)^{i+\frac{1}{2}}}
$$

Hence

$$
E\left(X^{k} \mid X>y\right)=y^{k-1} h(y, \lambda)+(k-1) E\left(X^{k-2} \mid X>y\right)+\frac{1}{\bar{F}(y, \lambda)} \alpha_{1}(y, \lambda) .
$$

Now for $k=2 r-1$, we have

$\alpha_{2}(y, \lambda)=\frac{\lambda}{2 \pi} \int_{y^{2}}^{\infty} z^{r-1-\frac{1}{2}} e^{-\frac{1}{2}\left(1+y^{2}\right) z} d z$

Integrating by parts we get

$$
\begin{aligned}
& \alpha_{2}(y, \lambda) \\
& =\left(\frac{k-2}{2}\right) ! \mu \phi\left(y \sqrt{1+\lambda^{2}}\right) \sum_{i=0}^{\frac{k-3}{2}} \frac{2^{i} y^{k-2-2 i}}{\left(\frac{k-2-2 i}{2}\right) !\left(1+\lambda^{2}\right)^{i+\frac{1}{2}}}+\mu \frac{k-2}{2} ! \frac{\Phi\left(-y \sqrt{1+\lambda^{2}}\right) 2^{\frac{k-1}{2}}}{\left(\sqrt{1+\lambda^{2}}\right)^{k-1\left(\frac{1}{2}\right) !}}
\end{aligned}
$$

Therefore,

$$
E\left(X^{k} \mid X>y\right)=y^{k-1} h(y, \lambda)+(k-1) E\left(X^{k-2} \mid X>y\right)+\frac{1}{\bar{F}(y, \lambda)} \alpha_{2}(y, \lambda) .
$$

Hence the result..

\section{Sufficiently:}

First when $=2 r$,

we have

$$
\frac{1}{\bar{F}(y, \lambda)} \int_{y}^{\infty} x^{k} f(x, \lambda) d x=y^{k-1} h(y, \lambda)+(k-1) E\left(X^{k-2} \mid X>y\right)+\frac{1}{F(y, \lambda)} \alpha_{1}(y, \lambda) .
$$

Or, after some maniposition 
Military Technical College

Kobry Elkobbah,

Cairo, Egypt

May 29-31,2012 $6^{\text {th }}$ International Conference on Mathematics and Engineering

Physics (ICMEP-6)

$\int_{y}^{\infty} x^{k} f(x, \lambda) d x=y^{k-1} f(y, \lambda)+\int_{y}^{\infty} x^{k-2} f(x, \lambda) d x+\alpha_{1}(y, \lambda)$

Differentiating both sides with respect to $y$, we obtain

$-y^{k} f(y, \lambda)=(k-1) y^{k-2} f(y, \lambda)+y^{k-1} 2 \lambda \phi(\lambda y) \phi(y)+y^{k-1} 2 \Phi(\lambda y) \phi(y)(-y)$

$+\left(\frac{k-2}{2}\right) ! \mu \phi\left(y \sqrt{1+\lambda^{2}}\right) \sum_{i=0}^{\frac{k-2}{2}} \frac{2^{i} y^{k-2-2 i}(-y)\left(1+\lambda^{2}\right)}{\left(\frac{k-2-2 i}{2}\right) !\left(1+\lambda^{2}\right)^{i+\frac{1}{2}}}$

$+\left(\frac{k-2}{2}\right) ! \mu \phi\left(y \sqrt{1+\lambda^{2}}\right) \sum_{i=0}^{\frac{k-2}{2}} \frac{2^{i} y^{k-3-2 i}(k-2-2 i)}{\left(\frac{k-2-2 i}{2}\right) !\left(1+\lambda^{2}\right)^{i+\frac{1}{2}}}$

which reduces to

$-y^{k} f(y, \lambda)=y^{k-1} 2 \Phi(\lambda y) \phi(y)(-y)$

Second when $k=2 r-1 \quad$,

we have

$\frac{1}{\bar{F}(y, \lambda)} \int_{y}^{\infty} x^{k} f(x, \lambda) d x=y^{k-1} h(y, \lambda)+(k-1) E\left(X^{k-2} \mid X>y\right)+\frac{1}{\bar{F}(y, \lambda)} \alpha_{2}(y, \lambda)$

Or,

$\int_{y}^{\infty} x^{k} f(x, \lambda) d x=y^{k-1} h(y, \lambda)+\int_{y}^{\infty} x^{k-2} f(x, \lambda) d x+\alpha_{2}(y, \lambda)$.

Differentiating both sides with respect to y, we obtain

$-y^{k} f(y, \lambda)=(k-1) y^{k-2} f(y, \lambda)+y^{k-1} 2 \lambda \phi(\lambda y) \phi(y)+y^{k-1} 2 \Phi(\lambda y) \phi(y)(-y)$

$+\left(\frac{k-2}{2}\right) ! \mu \phi\left(y \sqrt{1+\lambda^{2}}\right) \sum_{i=0}^{\frac{k-2}{2}} \frac{2^{i} y^{k-2-2 i}(-y)\left(1+\lambda^{2}\right)}{\left(\frac{k-2-2 i}{2}\right) !\left(1+\lambda^{2}\right)^{i+\frac{1}{2}}}$ 
Military Technical College

Kobry Elkobbah,

Cairo, Egypt

May 29-31,2012 $6^{\text {th }}$ International Conference on Mathematics and Engineering

Physics (ICMEP-6)

$+\left(\frac{k-2}{2}\right) ! \mu \phi\left(y \sqrt{1+\lambda^{2}}\right) \sum_{i=0}^{\frac{k-2}{2}} \frac{2^{i} y^{k-3-2 i}(k-2-2 i)}{\left(\frac{k-2-2 i}{2}\right) !\left(1+\lambda^{2}\right)^{i+\frac{1}{2}}}$

$+\mu\left(\frac{k-2}{2}\right) ! \frac{\phi\left(y \sqrt{1+\lambda^{2}}\right) 2^{\frac{k-1}{2}}\left(-\sqrt{1+\lambda^{2}}\right)}{\left(\sqrt{1+\lambda^{2}}\right)^{k-1}\left(\frac{-1}{2}\right) !}$

Which reduces to

$-y^{k} f(y, \lambda)=y^{k-1} 2 \Phi(\lambda y) \phi(y)(-y)$.

Or, $f(y, \lambda)=2 \Phi(\lambda y) \phi(y)$.

\section{Proof of theorem 2}

\section{Necessity}

we have

$$
\begin{aligned}
& E\left(X_{s i n}^{k} \mid X_{r i n}=x\right)=\int_{x}^{\infty} y^{k} \frac{f_{r, s i n}(x, y, \lambda)}{f_{r i n}(x, \lambda)} d y \\
& =\frac{C_{s i n}}{C_{r i n}}\left(\frac{\int_{x}^{\infty} y^{k} F(x, \lambda)^{r-1} f(x, \lambda)(F(y, \lambda)-F(x, \lambda))^{s-r-1} f(y, \lambda)(1-F(y, \lambda))^{n-s} d y}{F(x, \lambda)^{r-1} f(x, \lambda)(1-F(x, \lambda))^{n-r}}\right) \\
& =\frac{\frac{n !}{(r-1) !(s-r-1) !(n-s) !}}{\frac{n !}{(r-1) !(n-r) !}}\left(\frac{\int_{x}^{\infty} y^{k}(F(y, \lambda)-F(x, \lambda))^{s-r-1} f(y, \lambda)(1-F(y, \lambda))^{n-s} d y}{(1-F(x, \lambda))^{n-r}}\right) \\
& =\frac{(n-r) ! \int_{x}^{\infty} y^{k}(F(y, \lambda)-F(x, \lambda))^{s-r-1} f(y, \lambda)(1-F(y, \lambda))^{n-s} d y}{(1-F(x, \lambda))^{n-r}}
\end{aligned}
$$

Integrating by parts we get 
Military Technical College

Kobry Elkobbah,

Cairo, Egypt

May 29-31,2012 $6^{\text {th }}$ International Conference on Mathematics and Engineering

Physics (ICMEP-6)

$E\left(X_{s i n}^{k} \mid X_{r i n}=x\right)=x^{k}+\left[\frac{(n-r) !}{(1-F(x, \lambda))^{n-r}}\right] \times$

$\left[\sum_{j=r+1}^{s} k \int_{x}^{\infty} y^{k-1} \frac{\left\lfloor\left[\left(1-\int_{-\infty}^{y} \frac{1}{\pi} e^{-\frac{x^{2}}{2}} \int_{-\infty}^{\lambda x} e^{\left.-\frac{t 2}{2} d t d x\right)^{(n-j+1)}}\right] \beta_{1}(x, \lambda)\right\rfloor\right.}{(j-r-1) !(n-j+1) !} d y\right]$

$E\left(X_{s i n}^{k} \mid X_{r i n}=x\right)=x^{k}+\frac{(n-r) !}{(1-F(x, \lambda))^{n-r}} \eta_{s}(x, \lambda)$,

where

$\eta_{s}(x, \lambda)=\sum_{j=r+1}^{s} k \int_{x}^{\infty} y^{k-1} \frac{\left\lfloor\left[\left(1-\int_{-\infty}^{y} \frac{1}{\pi} e^{-\frac{x^{2}}{2}} \int_{-\infty}^{\lambda x} e^{-\frac{t^{2}}{2}} d t d x\right)^{(n-j+1)}\right] \psi_{1}(x, y, \lambda) \mid\right.}{(j-r-1) !(n-j+1) !} d y_{,}$

and

$\psi_{1}(x, y, \lambda)=\left[\left(\int_{-\infty}^{y} \frac{1}{\pi} e^{-\frac{x^{2}}{2}} \int_{-\infty}^{\lambda x} e^{-\frac{t^{2}}{2}} d t d x-\int_{-\infty}^{x} \frac{1}{\pi} e^{-\frac{u^{2}}{2}} \int_{-\infty}^{\lambda u} e^{-\frac{t^{2}}{2}} d t d u\right)^{(j-r-1)}\right]$.

which complete the proof.

Sufficiently:

Assume that $E\left(X_{s: n}^{k} \mid X_{r: n}=x\right)=x^{k}+\frac{1}{(1-F(x, \lambda))^{n-F}} \eta_{s}(x, \lambda)$.

$\frac{(n-r) !}{(s-r-1) !(n-s) !}\left(\frac{\int_{x}^{\infty} y^{k}(F(y, \lambda)-F(x, \lambda))^{s-r-1} f(y, \lambda)(1-F(y, \lambda))^{n-s} d y}{(1-F(x, \lambda))^{n-r}}\right)=$

$x^{k}+\frac{1}{(1-F(x, \lambda))^{n-r}} \eta_{s}(x, \lambda)$

Multiplying both sides by $(1-F(x, \lambda))^{(n-r)}$, we obtain 
Military Technical College

Kobry Elkobbah,

Cairo, Egypt

May 29-31,2012 $6^{\text {th }}$ International Conference on Mathematics and Engineering

Physics (ICMEP-6)

$\frac{(n-r) !}{(s-r-1) !(n-s) !}\left(\int_{x}^{\infty} y^{k}(F(y, \lambda)-F(x, \lambda))^{s-r-1} f(y, \lambda)(1-F(y, \lambda))^{n-s} d y\right)=$

$x^{k}(1-F(x, \lambda))^{n-r}+\eta_{s}(x, \lambda)$.

Differentiating both sides with respect to $x$, we obtain

$$
\begin{aligned}
& \frac{-(n-r) !}{(s-r-1) !(n-s) !}\left[x^{k}(F(x, \lambda)-F(x, \lambda))^{s-r-1} f(x, \lambda)(1-F(x, \lambda))^{n-s}\right] \\
& -\frac{(n-r) !(s-r-1)}{(s-r-1) !(n-s) !}\left(\int_{x}^{\infty} y^{k}(F(y, \lambda)-F(x, \lambda))^{s-r-2} f(x, \lambda) f(y, \lambda)(1-F(y, \lambda))^{n-s} d y\right) \\
& =k x^{k-1}(1-F(x, \lambda))^{n-r}-(n-r) x^{k}(1-F(x, \lambda))^{n-r-1} f(x, \lambda) \\
& \left.-(n-r) ! \sum_{j=r+1}^{s} k x^{k-1} \frac{\left[\mid\left(1-\int_{-\infty}^{x} \frac{1}{\pi} e^{\left.\left.-\frac{u^{2}}{2} \int_{-\infty}^{\lambda u} e^{-\frac{t^{2}}{2}} d t d u\right)^{(n+j+1)}\right]} \beta_{1}(x, \lambda) \mid\right.\right.}{(j-r-1) !(n-j+1) !}\right] \\
& -(n-r) ! \sum_{j=r+2}^{s} \int_{x}^{\infty} \int^{k-1} \frac{\left[\left(1-\int_{-\infty}^{y} \frac{1}{\pi} e^{-\frac{x^{2}}{2}} \int_{-\infty}^{\lambda x} e^{-\frac{t^{2}}{2}} d t d x\right)^{(n-j+1)}\right]}{(j-r-2) !(n-j+1) !} \psi_{2}(x, y, \lambda) \mid
\end{aligned}
$$

Where $\psi_{2}(x, y, \lambda)=\left[\left(\int_{-\infty}^{y} \frac{1}{\pi} e^{-\frac{x^{2}}{2}} \int_{-\infty}^{\lambda x} e^{-\frac{t^{2}}{2}} d t d x-\int_{-\infty}^{x} \frac{1}{\pi} e^{-\frac{u^{2}}{2}} \int_{-\infty}^{\lambda u} e^{-\frac{t^{2}}{2}} d t d u\right)^{(j-r-2)}\right]$

$$
\begin{aligned}
& -\frac{(n-r) !}{(8-r-2) !(n-s) !}\left(\int_{x}^{\infty} y^{k}(F(y, \lambda)-F(x, \lambda))^{s-r-2} f(y, \lambda)(1-F(y, \lambda))^{n-s} d y\right) \\
& =-(n-r) x^{k}(1-F(x, \lambda))^{n-r-1}
\end{aligned}
$$


Military Technical College Kobry Elkobbah,

Cairo, Egypt

May 29-31,2012

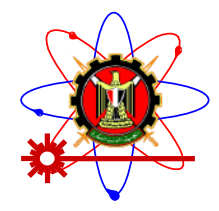

$6^{\text {th }}$ International Conference on Mathematics and Engineering

Physics (ICMEP-6)

$-(n-r) ! \sum_{j=r+2}^{s} k \int_{x}^{\infty} y^{k-1} \frac{\left|\left[\left(1-\int_{-\infty}^{y} \frac{1}{\pi} e^{-\frac{x^{2}}{2}} \int_{-\infty}^{\lambda x} e^{-\frac{t^{2}}{2}} d t d x\right)^{(n-j+1)}\right] \psi_{2}(x, y, \lambda)\right|}{(j-r-2) !(n-j+1) !} d y$.

Now, let $I_{4}(x, \lambda)=\int_{x}^{\infty} y^{k}(F(y, \lambda)-F(x, \lambda))^{s-r-2} f(y, \lambda)(1-F(y, \lambda))^{n s} d y$.

Integrating by parts, gives

$$
\begin{aligned}
& I_{4}(x, \lambda)=x^{k}(n-s) !(s-r-2) !(1-F(x, \lambda))^{n-r-1} \\
& +\int_{x}^{\infty} k y^{k-1}(n-s) !(s-r-2) !\left[\sum_{j=r+2}^{s} \frac{(F(y, \lambda)-F(x, \lambda))^{j-r-2}(1-F(y, \lambda))^{n-j+1}}{(j-r-2) !(n-j+1) 1}\right] d y . \\
& (n-r) ! x^{k}(1-F(x, \lambda))^{n-r-1}+ \\
& \int_{x}^{\infty} k y^{k-1}(n-r) !\left[\sum_{j=r+2}^{s} \frac{(F(y, \lambda)-F(x, \lambda))^{j-r-2}(1-F(y, h))^{n-j+1}}{(j-r-2) !(n-j+1) !}\right] d y \\
& =(n-r) x^{k}(1-F(x, \lambda))^{n-r-1} \\
& +(n-r) ! \sum_{j=r+2}^{s} k \int_{x}^{\infty} y^{k-1} \frac{\left|\left[\left(1-\int_{-\infty}^{y} \frac{1}{\pi} e^{-\frac{x^{2}}{2}} \int_{-\infty}^{\lambda x} e^{-\frac{t^{2}}{2}} d t d x\right)^{(n-j+1)}\right] \psi_{2}(x, y, \lambda)\right|}{(j-r-2) !(n-j+1) !} d y . \\
& \text { Where } \psi_{2}(x, y, \lambda)=\left[\left(\int_{-\infty}^{y} \frac{1}{\pi} e^{-\frac{x^{2}}{2}} \int_{-\infty}^{\lambda x} e^{-\frac{t^{2}}{2}} d t d x-\int_{-\infty}^{x} \frac{1}{\pi} e^{-\frac{u^{2}}{2}} \int_{-\infty}^{\lambda u} e^{-\frac{t^{2}}{2}} d t d u\right)^{(j-r-2)}\right] \text {, } \\
& \text { Or, } \int_{x}^{\infty} k y^{k-1}(n-r) !\left\lfloor\sum_{j=r+2}^{s} \frac{(F(y, \lambda)-F(x, \lambda))^{j-r-2}(1-F(y, \lambda))^{n-j+1}}{(j-r-2) !(n-j+1) !}\right\rfloor d y= \\
& (n-r) ! \sum_{j=r+2}^{s} k \int_{x}^{\infty} y^{k-1} \frac{\left\lfloor\left[\left(1-\int_{-\infty}^{y} \frac{1}{\pi} e^{-\frac{x^{2}}{2}} \int_{-\infty}^{\lambda x} e^{-\frac{t^{2}}{2}} d t d x\right)^{(n-j+1)}\right] \psi_{2}(x, y, \lambda)\right\rfloor}{(j-r-2) !(n-j+1) !} d y
\end{aligned}
$$


Military Technical College

Kobry Elkobbah,

Cairo, Egypt

May 29-31,2012 $6^{\text {th }}$ International Conference on Mathematics and Engineering

Physics (ICMEP-6)

Where $\psi_{2}(x, y, \lambda)=\left[\left(\int_{-\infty}^{y} \frac{1}{\pi} e^{-\frac{x^{2}}{2}} \int_{-\infty}^{\lambda x} e^{-\frac{t^{2}}{2}} d t d x-\int_{-\infty}^{x} \frac{1}{\pi} e^{-\frac{u^{2}}{2}} \int_{-\infty}^{\lambda u} e^{-\frac{t^{2}}{2}} d t d u\right)^{(j-r-2)}\right]$.

Finally, this implies that

$F(x, \lambda)=\int_{-\infty}^{x} \frac{1}{\pi} e^{-\frac{x^{2}}{2}} \int_{-\infty}^{\lambda x} e^{-\frac{t^{2}}{2}} d t d x$

Which is the cdf of the skew normal distribution with parameter $\lambda$.

\section{Proof. Theorem 3}

\section{Necessity.}

We have

$$
\begin{aligned}
& E\left(X_{s i n, \bar{m}, k}^{k} \mid X_{r: m, \bar{m}, k}=x\right)=\int_{x}^{\infty} y^{k} \frac{f_{r, s i n, \bar{m}, k}(x, y, \lambda)}{f_{r i n, \bar{m}, k}(x, \lambda)} d y \\
& =\frac{d_{s-1}}{d_{r-1}} \frac{\int_{x}^{\infty} y^{k}\left[(1-F(x, \lambda))^{m+1}-(1-F(y, \lambda))^{m+1}\right]^{s-r-1} f(y, \lambda)(1-F(y, \lambda))^{(m+1)(n-s)+k-1} d y}{(m+1)^{s-r-1}(s-r-1) !(1-F(x, \lambda))^{(m+1)(n-r-1)+k}},
\end{aligned}
$$

Integrating by parts we get

$$
\begin{aligned}
& E\left(X_{s i n, \overline{m_{1}, k}}^{k} \mid X_{r_{m} n_{2}, \overline{m_{2}, k}}=x\right)=x^{k} \\
& +\left(\frac{(m+1)(n-s-1)+k}{m+1}\right) !\left[\frac{(m+1)^{s-r} \prod_{j=r+1}^{s}[(m+1)(n-j)+k]}{(1-F(x, \lambda))^{(m+1)(n-r-1)+k}}\right] \times
\end{aligned}
$$

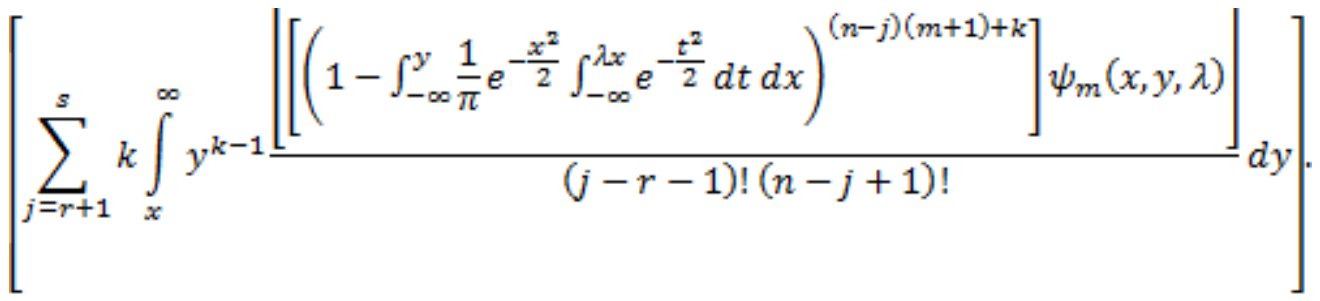

$$
\begin{aligned}
& E\left(X_{s i n}^{k} \mid X_{r i n}=x\right)=
\end{aligned}
$$


Military Technical College

Kobry Elkobbah,

Cairo, Egypt

May 29-31,2012 $6^{\text {th }}$ International Conference on Mathematics and Engineering

Physics (ICMEP-6)

$$
x^{k}+\left(\frac{(m+1)(n-s-1)+k}{m+1}\right) ! \frac{(m+1)^{s-r} \prod_{j=r+1}^{s}\lfloor(m+1)(n-j)+k\rfloor}{(1-F(x, \lambda))^{(m+1)(n-r-1)+k}} \gamma_{s}(x, \lambda),
$$

where

$\gamma_{s}(x, \lambda)=\sum_{j=r+1}^{s} k \int_{x}^{\infty} y^{k-1} \frac{\left\lfloor\left[\left(1-\int_{-\infty}^{y} \frac{1}{\pi} e^{-\frac{x^{2}}{2}} \int_{-\infty}^{\lambda x} e^{-\frac{t^{2}}{2}} d t d x\right)^{(m+1)(n-j)+k}\right] \psi_{1}(x, y, \lambda) \mid\right.}{\left(\frac{[(m+1)(n-j)+k]}{(m+1)}\right) !(j-r-1) !}$

and

$$
\psi_{m}(x, y, \lambda)=
$$

$$
\left[\left(1-\int_{-\infty}^{x} \frac{1}{\pi} e^{-\frac{u^{2}}{2}} \int_{-\infty}^{\lambda u} e^{-\frac{t^{2}}{2}} d t d u\right)^{m+1}-\left(1-\int_{-\infty}^{y} \frac{1}{\pi} e^{-\frac{x^{2}}{2}} \int_{-\infty}^{\lambda x} e^{-\frac{t^{2}}{2}} d t d x\right)^{m+1}\right]^{(j-r-1)}
$$

which proves necessity.

\section{Sufficiently:}

Assume that

$$
\begin{aligned}
& E\left(X_{s+n}^{k} \mid X_{r i n}=x\right)=x^{k}+ \\
& \left(\frac{(m+1)(n-s-1)+k}{m+1}\right) ! \frac{(m+1)^{s-r} \prod_{j=r+1}^{s}\lfloor(m+1)(n-j)+k\rfloor}{(1-F(x, \lambda))^{(m+1)(n-r-1)+k}} \gamma_{s}(x, \lambda) . \\
& \frac{d_{s-1}}{d_{r-1}} \frac{\int_{x}^{\infty} y^{k}\left[(1-F(x, \lambda))^{m+1}-(1-F(y, \lambda))^{m+1}\right]^{s-r-1} f(y, \lambda)(1-F(y, \lambda))^{(m+1)(n-s)+k-1} d y}{(m+1)^{s-r-1}(s-r-1) !(1-F(x, \lambda))^{(m+1)(n-r-1)+k}} \\
& =x^{k}+\left(\frac{(m+1)(n-s-1)+1}{m+1}\right) ! \frac{(m+1)^{s-r} \prod_{j=r+1}^{s}\lfloor(m+1)(n-j)+k\rfloor}{(1-F(x, \lambda))^{(m+1)(n-r-1)+k}} \gamma_{s}(x, \lambda) .
\end{aligned}
$$

Multiplying both sides by $(1-F(x, \lambda))^{(m+1)(n-r-1)+k}$, we obtain 
Military Technical College Kobry Elkobbah,

Cairo, Egypt

May 29-31,2012 $6^{\text {th }}$ International Conference on Mathematics and Engineering

Physics (ICMEP-6)

$$
\begin{aligned}
& \frac{d_{s-1}}{d_{r-1}} \frac{\int_{x}^{\infty} y^{k}\left[(1-F(x, \lambda))^{m+1}-(1-F(y, \lambda))^{m+1}\right]^{s-r-1} f(y, \lambda)(1-F(y, \lambda))^{(m+1)(n-s)+k-1} d y}{(m+1)^{s-r-1}(s-r-1) !} \\
& =x^{k}(1-F(x, \lambda))^{(m+1)(n-r-1)+k}+ \\
& \left(\frac{(m+1)(n-s-1)+k}{m+1}\right) !(m+1)^{s-r} \prod_{j=r+1}^{s}[(m+1)(n-j)+k] \gamma_{s}(x, \lambda) .
\end{aligned}
$$

Now, differentiating both sides with respect to $x$, we get

$$
\begin{aligned}
& \frac{d_{s-1}}{d_{r-1}} \frac{\int_{x}^{\infty} y^{k}\left[(1-F(x, \lambda))^{m+1}-(1-F(y, \lambda))^{m+1}\right]^{s-r-1} f(y, \lambda)(1-F(y, \lambda))^{(m+1)(n-s)+k-1} d y}{(m+1)^{s-r-1}(s-r-1) !} \\
& \times(m+1)(1-F(x, \lambda))^{m}(-f(x, \lambda))=k x^{k-1}(1-F(x, \lambda))^{(m+1)(n-r-1)+k}+ \\
& x^{k}[(m+1)(n-r-1)+k](-f(x, \lambda))(1-F(x, \lambda))^{(m+1)(n-r-1)+k}+ \\
& \sum_{j=r+1}^{s} \int_{x}^{\infty} y^{k-1} \frac{\left[\left(1-\int_{-\infty}^{y} \frac{1}{\pi} e^{-\frac{x^{2}}{2}} \int_{-\infty}^{\lambda x} e^{-\frac{t^{2}}{2}} d t d x\right)^{(m+1)(n-j)+k}\right]}{\left(\frac{[(m+1)(n-j)+k]}{(m+1)}\right) !(j-r-1) !} \times \\
& (j-r-1)(m+1)\left(\frac{-1}{\pi} e^{-\frac{x^{2}}{2}} \int_{-\infty}^{\lambda x} e^{-\frac{t^{x}}{2}} d t\right)\left(1-\int_{-\infty}^{x} \frac{1}{\pi} e^{-\frac{u^{2}}{2}} \int_{-\infty}^{\lambda u} e^{-\frac{t^{2}}{2}} d t d u\right)^{m} \times \\
& {\left[\left(1-\int_{-\infty}^{x} \frac{1}{\pi} e^{-\frac{u^{2}}{2}} \int_{-\infty}^{\lambda u} e^{-\frac{t^{x}}{2}} d t d u\right)^{m+1}-\left(1-\int_{-\infty}^{y} \frac{1}{\pi} e^{-\frac{x^{2}}{2}} \int_{-\infty}^{\lambda u} e^{-\frac{t^{x}}{2}} d t d u\right)^{m+1}\right]^{(j-r)}-} \\
& k x^{k-1}(1-F(x, \lambda))^{(m+1)(n-r-1)+k}-
\end{aligned}
$$

Now let

$$
I_{5}=\int_{x}^{\infty} y^{k}\left[(1-F(x, \lambda))^{m+1}-1-F(y, \lambda)^{m+1}\right]^{s-r-1} f(y, \lambda)(1-F(y, \lambda))^{(m+1)(n-s)+k-1} d y
$$

Integrating by parts, we get 
Military Technical College

Kobry Elkobbah,

Cairo, Egypt

May 29-31,2012

$F(x, \lambda)=\int_{-\infty}^{x} \frac{1}{\pi} e^{-\frac{x^{2}}{2}} \int_{-\infty}^{\lambda x} e^{-\frac{t^{2}}{2}} d t d x$

Which is the cdf of the skew normal distribution with parameter $\lambda$.

This completes the proof theorem 3 . $6^{\text {th }}$ International Conference on Mathematics and Engineering Physics (ICMEP-6)

\section{References}

- Arnold, B. C. , Balakrishnan, N. and Nagarajua, H. N. (2008). A first course in order statistics, John Wiely \& Sons, New York.

- Balakrishnan, N. (1988). Recurrence relations for order statistics from $n$ independent and non-identical distributed random variables, Ann. Inst. Statist. Math. 40,273-277.

- Balakrishnan, N. and Cohen, A. C. (1991). Order statistics and inference : Estimation methods. San Diego : Academic Press.

- David, H. A. and Nagaraja, H. N. (2003). Order statistics, 3rd ed. , WileyInterscience [John Wiley \&Sons], Hoboken, NJ,.

- Huang, J. S. (1989). Moment problem of order statistics: A review, Inter. Statist. Rev., Vol. 57, pp. 59-66,

- Huang, J. S. and Lin, G. D. (1984). Characterizations of distributions by linear combinations of order statistics, Bull. Inst. Math., Academia Sincia, Vol. 12, pp. 179-202.

- Khan, A. H. and Khan, I. A. (1987). Moments of order statistics from Burr XII distribution and its characterization, Metron XLV-N1-2, 30, 21-29.

- Azzalini A.(1985). A class of distributions which includes the normal ones. Scand J Stat , 12:171 178 .

- O'Hagan, A. and Leonard, T. (1976). Bayes estimation subject to uncer- tainty about parameter constraints. Biometrika, 63, 201-203.

- Henze NA (1986). A probabilistic representation of the skew-normal distrib- ution. Scand J Stat 13:271 275.

- Azzalini A, Dalla Valle A (1996). The multivariate skew-normal distribu- tion. Biometrika 83:715 726.

- Branco M, DeyDK (2001). Ageneral class ofmultivariate elliptical distribu- tion. JMultivar Anal 79:99 113.

- Loperfido N (2001). Quadratic forms of skew-normal random vectors. Stat Probab Lett 54:381 387. 
Military Technical College

Kobry Elkobbah,

Cairo, Egypt

May 29-31,2012

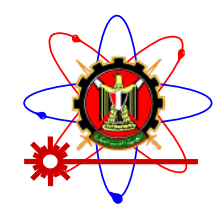

- Arnold BC, Beaver RJ (2002). Skewmultivariate models related to hidden truncation and/or selective reporting. Test 11:7 54 .

- Balakrishnan N (2002). Discussion on Skew multivariate models related to hidden truncation and/or selective reporting by B.C. Arnold and R.J. Beaver. Test 11:37 39.

- Azzalini A, Chiogna M (2004). Some results on the stress-strength model for skew normal variate. Metron LXII:315 326.

- Lin, G. D.(1989). The product moments of order statistics with applications to characterizations of distributions, J. Statist. Plan. Infer., Vol 21, pp.395-406 